\title{
Case \\ Report \\ Leiomyosarcoma of the maxilla as second malignancy in retinoblastoma
}

\author{
Qureshi SS ${ }^{1}$, Mistry RC ${ }^{2}$, Natrajan ${ }^{3}$, Gujral S4, Laskar S5, Banavali S \\ 1,2,3 Department of Surgical Oncology, ${ }^{4}$ Department of Pathology, 5,6 Department of Pediatric Oncology, \\ Tata Memorial Hospital, Ernest Borges Road, Parel, Bombay, India.
}

Correspondence to: Dr. Sajid S Qureshi, E-mail: sajidshafique@rediffmail.com

\begin{abstract}
Patients with hereditary retinoblastoma are at increased risk of second primary tumor, the commonest tumor being osteosarcoma. Leiomyosarcoma developing as second primary neoplasm in retinoblastoma patients is unusual and most have occurred in the field of previous radiotherapy. Although with aggressive therapy better survival can be achieved, the overall prognosis of patients developing these second neoplasms is poor. In this report we present a case of leiomyosarcoma of the maxilla as a second neoplasm in a patient with bilateral retinoblastoma which has developed outside the radiation field.
\end{abstract}

Key words: Late effects, retinoblastoma, sarcoma

\section{Introduction}

Second malignant neoplasm represents the primary cause of deaths in patients with hereditary retinoblastoma. ${ }^{[1]}$ The reported risk varies widely, but a cumulative incidence of $1 \%$ for each year of life has been suggested as an approximate estimate. ${ }^{[2]}$ Leiomyosarcomas (LMS) of the head and neck are rare. The last review of the literature made in 1995 by Izumi et $\mathrm{al}^{[3]}$ identified 59 cases of oral LMS. Subsequently sporadic reports of LMS of the maxilla have been published in the literature. ${ }^{[4-7]}$ Like other soft tissue sarcomas most LMS in survivors of retinoblastoma develop within the field of radiation. Very few cases have been reported at distant places. ${ }^{[2,8-9]}$

We report a case of LMS of the maxilla arising as a second neoplasm in a patient with bilateral retinoblastoma, which has developed outside the radiation field and followed a rapid fulminant course.

\section{Case Report}

A 15-year-old boy presented with a two-month history of painful swelling in the right cheek. At the age of one and a half years, he had been diagnosed as having bilateral retinoblastoma and was treated with enucleation of right eye and radiotherapy (RT) to the left orbit by a lateral field with Cobalt 60 gamma rays. A total dose of $36 \mathrm{~Gy}$ in 9 fractions over 27 days at 4 Gy per fraction (alternate day radiation) was administered.

Physical examination revealed a unilateral bulge in the hard palate and gingivo buccal sulcus. A biopsy revealed spindle cell sarcoma of intermediate grade. Computerized tomogram scan showed a large mass in the maxillary antrum destroying the maxillary alveolus and the posterolateral wall with a superior extension into the orbit. At surgery the lesion was found to be invading the anterior wall of maxillary antrum and extending into the nasal cavity, infratemporal fossa, and ethmoid. A total maxillectomy was performed but tumor free margins could not be achieved due to disease infiltrating the infratemporal fossa. Postoperatively histopathological examination revealed a maxillary LMS with involvement of the ethmoid. The tumor was largely submucosal and had spindle-shaped cells with marked pleomorphism and brisk mitotic activity [Figure 1]. Immunohistochemistry showed tumor cell to be positive for vimentin and smooth muscle actin (SMA) [Figure 2] and negative for S100 and LCA. After three weeks there was rapid growth of the residual disease in the maxillary fossa. In view of the aggressive nature of the disease palliative RT and cisplatin based chemotherapy was given. The 


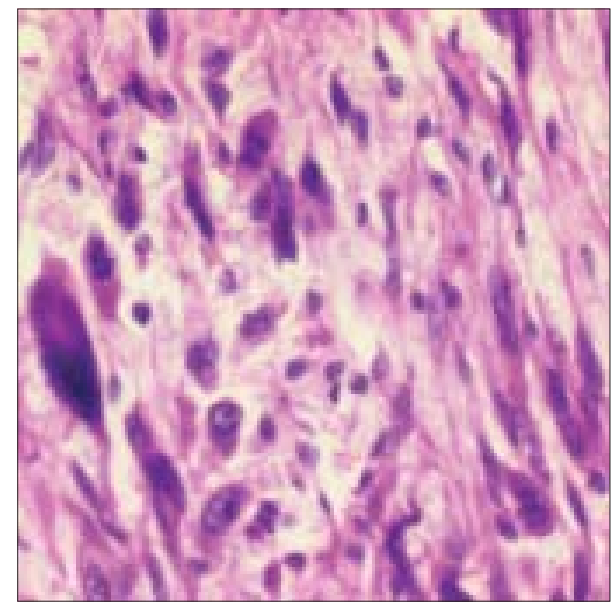

Figure 1: High power view showing spindle shaped tumor cells with pleomorphism and mitosis (H\&E staining, 40x)

patient initially had good subjective response but died after three months from progressive tumor.

\section{Discussion}

Long-term survival in patients with hereditary retinoblastoma is significantly reduced because of the risk of second malignant neoplasm. ${ }^{[1]}$ The cumulative incidence of second cancers after retinoblastoma reported in the literature, ranges from $8.4 \%$ at 18 years after diagnosis to $90 \%$ after 30 years. ${ }^{[10]}$ The risk factors for second non-ocular tumors occurring after retinoblastoma includes genetic predisposition in patients with hereditary retinoblastoma. Patients treated for hereditary retinoblastoma are at an increased risk to develop non-ocular malignancies due to a mutation in the second RB1 allele in different tissues. ${ }^{[1]}$ Radiation boosts the already high rate of second malignancy in retinoblastoma. Chemotherapy containing alkylating agents, alone or in combination with radiotherapy, also seems to be involved in the development of second cancers. ${ }^{[1]}$ Thirty-five different histological types of second malignant neoplasm have been reported in treated retinoblastoma patients. ${ }^{[10]}$ O steogenic sarcomas are the predominant type identified.

LMS of maxilla as second malignant neoplasm in retinoblastoma usually arises within the radiation field. Clinically most oral cavity LMS are in the jaw bones $(68 \%)$, predominantly in the maxilla $(47 \%)$. ${ }^{[3]}$ The age of the patient's ranges from 10 months to 88 years and a slight male predilection is seen. The usual clinical features are a painless, slow growing, and discreet swelling usually without ulceration on the upper jaw. ${ }^{[3]}$

$\mathrm{H}$ istology shows fusiform cells with intracellular myofibrils and blunt ended nuclei, which are aligned

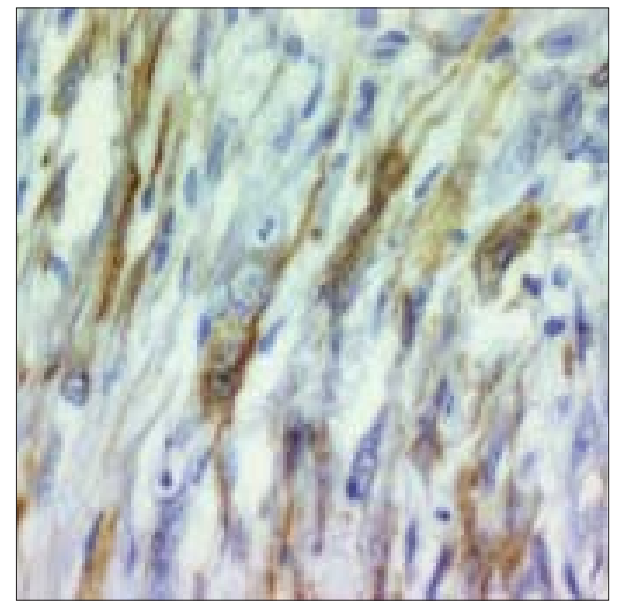

Figure 2: Immunohistochemistry showing tumor cells positive for SMA (H\&E staining, 40x)

in a palisade pattern. On light microscopy it is at times impossible to differentiate a LMS from a fibrosarcoma, malignant fibrous histiocytoma and neurogenic sarcomas. ${ }^{[3-5]}$ I mmunohistochemical staining to SMA and desmin may facilitate diagnosis. U Itrastructural examination by electron microscopy also confirms smooth muscle origin of tumor. ${ }^{[3]}$

Treatment of LMS is primarily a wide local excision. ${ }^{[3-4]}$ The tumors are usually not well encapsulated making it difficult to get uniform wide margins. These tumors are in addition resistant to chemotherapy and $\mathrm{RT}{ }^{[3]}$ Some centers have tried adjuvant chemotherapy with adriamycin alone, adriamycin with cyclophosphamide or dacarbazine. ${ }^{[4]}$ A $50 \%$ rate of local recurrence and distant metastasis to the lung, liver and regional lymph nodes is reported. ${ }^{[4]}$ The high incidence of local recurrence even after a wide excision with adequate margins point towards the aggressive nature of the disease. Prognosis of this tumor is poor in most cases with a five-year disease free survival of $23 \%$. $^{[4]}$

In the present case the second neoplasm developed after a latency period of 13 years. We believe that the lesion appeared outside the defined field of radiation although the possibility of scatter effect may not be completely excluded. Achievement of adequate surgical margin was impractical due to invasion into the adjacent structures and there was rapid growth of residual disease after three weeks of surgery, which was not controlled with RT and chemotherapy. The patient had a progressive fulminant course and died after three months.

Although, the long-term cumulative incidence of second tumors in retinoblastoma will never be zero because of the genetic predisposition of these patients to develop second cancers, conservative ophthalmologic treatments, 
brachytherapy and limited adjuvant chemotherapy after enucleation can be considered in an attempt to reduce the risk of second cancers. ${ }^{[10]}$ The prognosis of these neoplasm's' can be improved by aggressive treatment, but currently still remains poor. Therefore it is imperative to diagnose the second cancers early for which the parents and patients need to be counseled to seeking early medical advice and the physicians have to ensure that a careful and long-term follow-up of these patients is maintained.

\section{REFERENCES}

1. Aerts I, Pacquement H, Doz F, Mosseri V, Desjardins L, Sastre X, et al. Outcome of second malignancies after retinoblastoma: $\mathrm{A}$ retrospective analysis of 25 patients treated at the Institut Curie. Eur J Cancer 2004;40: 1522-9

2. Venkatraman L, Goepel JR, Steele K, Dobbs SP, Lyness RW, McCluggage WG. Soft tissue, pelvic, and urinary bladder leiomyosarcoma as second neoplasm following hereditary retinoblastoma. J Clin Pathol 2003;56:233-6.

3. Izumi K, Maeda T, Cheng J, Saku T. Primary leiomyosarcoma of the maxilla with regional lymph node metastasis: Report of a case and review of the literature. Oral Surg Oral Med Oral Med Oral Radiol
Endod 1995;80:310-9.

4. Sumida T, Hamakawa H, Otsuka K, Tanioka H. Leiomyosarcoma of the maxillary sinus with cervical lymph node metastases. J Oral Maxillofac Surg 2001;59:568-71.

5. Savastano G, Palombini L, Muscariello V, Erra S. Leiomyosarcoma of the maxilla: A case report. J Oral Maxillofac Surg 1998;56:1101-3.

6. Wada S, Yue L, Furuta I, Takazakura T. Leiomyosarcoma in the maxilla: A case report. Int J Oral Maxillofac Surg 2002;31:219-21.

7. Sedghizadeh PP, Angiero F, Allen CM, Kalmar JR, Rawal Y, Albright EA. Post-irradiation leiomyosarcoma of the maxilla: Report of a case in a patient with prior radiation treatment for retinoblastoma. Oral Surg Oral Med Oral Pathol Endod 2004;97:726-31.

8. Bleoo SL, Godbout R, Rayner D, Tamimi Y, Moore RB. Leiomyosarcoma of the bladder in a retinoblastoma patient. Urol Int 2003;71:118-21.

9. Ryan RS, Gee R, O'Connell JX, Harris AC, Munk PL. Leiomyosarcoma of the distal femur in a patient with a history of bilateral retinoblastoma: A case report and review of the literature. Skeletal Radiol 2003;32:476-80.

10. Dunkel IJ, Gerald WL, Roesenfield NS, Strong EW, Abramson DH, Ghavimi F. Outcome of patients with a history of bilateral retinoblastoma treated for a second malignancy: the Memorial Sloan-Kettering experience. Med Pediatr Oncol 1998;30:59-62.

Source of Support: Nil, Conflict of Interest: None declared.

\section{Author Help: Sending a revised article}

1) Include the referees' remarks and point to point clarification to those remarks at the beginning in the revised article file its elf. In addition, mark the changes as underlined or coloured text in the article. Please include in a single file

a. referees' comments

b. point to point clarifications on the comments

c. revised article with text highlighting the changes done

2) Include the original comments of the review ers/editor w ith point to point reply at the beginning of the article in the 'Article File'. To ensure that the review er can assess the revised paper in timely fashion, please reply to the comments of the referees/editors in the following manner.

- There is no data on follow-up of these patients.

Authors' Reply: The follow up of patients have been included in the results section [Page 3, para 2]

- Authors should highlight the relation of complication to duration of diabetes.

Authors' Reply: The complications as seen in our study group has been included in the results section [Page 4, Table] 